\title{
Correction to: Two-season agriculture and irrigated rice during the Dian: radiocarbon dates and archaeobotanical remains from Dayingzhuang, Yunnan, Southwest China
}

\author{
Rita Dal Martello ${ }^{1}$ Xiaorui $\mathrm{Li}^{2} \cdot$ Dorian Q. Fuller ${ }^{1,3,4}$ \\ Published online: 8 May 2021 \\ (C) Springer-Verlag GmbH Germany, part of Springer Nature 2021
}

Correction to: Archaeological and Anthropological Sciences (2021) 13:62 https://doi.org/10.1007/s12520-020-01268-y

The original version of this paper was published with error in author's given and family names: "Dal Martello Rita, Li Xiaorui, Dorian Q. Fuller". Authors apologize for making an error in the presentation of the name of the authors.

The corrected author presentation is "Rita Dal Martello, Xiaorui Li, Dorian Q. Fuller".

This mix between family name and given name of Rita Dal Martello and Xiaorui Li happened during the final editing of the manuscript and passed unnoticed during submission and revision. We like to apologize for this mistake and thank Archaeological and Anthropological Sciences Editor-inChief and journal manager for giving us the chance to correct this error.

Publisher's note Springer Nature remains neutral with regard to jurisdictional claims in published maps and institutional affiliations.

The online version of the original article can be found at https://oi.org/ 10.1007/s12520-020-01268-y

Dorian Q. Fuller

d.fuller@ucl.ac.uk

1 Department of Archaeology, Max Planck Institute for the Science of Human History, Kahlaische Strasse 10, 07745 Jena, Germany

2 Yunnan Province Institute of Cultural Relics and Archaeology, Kunming 650118, China

3 Institute of Archaeology, University College London, 31-34 Gordon Square, London WC1H OPY, UK

4 School of Cultural Heritage, Northwest University, Xi'an 710069 , China 